\title{
Estratégias de capacitação de professores do ensino fundamental em atividade física e alimentação saudável
}

\author{
Arilson Fernandes Mendonça de Sousa ${ }^{1}$ \\ Júlia Aparecida Devidé Nogueira ${ }^{2}$ \\ Alexandre Luiz Gonçalves de Rezende ${ }^{2}$ \\ ${ }^{1}$ Mestre em Educação Física, Faculdade de Educação Física, Universidade de Brasília, DF, Brasil \\ ${ }_{2}^{2}$ Professor Adjunto, Faculdade de Educação Física, Universidade de Brasília, DF, Brasil
}

\begin{abstract}
Resumo: $O$ estudo avaliou efeitos de aprendizagem gerados por intervenções educativas longa (IL) e curta (IC) nos conhecimentos em alimentação saudável e atividade física. 42 professores de duas escolas públicas de ensino fundamental responderam a questionários de conhecimento com nota de 0 a 10 , pré e pós-intervenção. A IL ofereceu um curso de 40 horas e a IC ofereceu uma palestra de 30 minutos; os dois grupos receberam o mesmo material educativo impresso. As intervenções produziram aumento estatisticamente significativo $(p<0,05)$ no conhecimento médio em alimentação saudável $[\mathrm{IL}:+1,19$ ( $E P=0,31)$ e IC: $+1,18(E P=0,34)$ pontos]. Em atividade física a mudança no conhecimento médio só foi estatisticamente significativa na IL [IL $+1,69(E P=0,52)$ e IC $-0,36$; $(E P=0,45)$ pontos]. Houve diferença significativa nas notas finais entre grupos para conhecimento em atividade física. Ações educativas simples produziram efeitos positivos no conhecimento sobre alimentação saudável, entretanto, para atividade física pode ser necessário maior tempo de intervenção.
\end{abstract}

Palavras-chave: Atividade física. Alimentação saudável. Educação. Escola.

\section{Training strategies for elementary education teachers in physical activity and health eating}

Abstract: The study evaluated the learning effects generated by long (IL) and short (IC) educational interventions in health eating and physical activity knowledge. 42 teachers from two public elementary schools completed knowledge questionnaires with a 0-10 grade, pre and post-intervention. IL offered a 40 hours course and IC offered a 30 minutes lecture, and both groups received the same printed educational material. The intervention produced a statistically significant $(p<0.05)$ increased in averaged health eating knowledge [IL: +1.19 (SE=0.31) and $\mathrm{Cl}:+1.18$ (SE 0.34) points]. In physical activity, change in average knowledge was only statically significant in IL [IL + $1.69(\mathrm{SE}=0.52)$ and IC -0.36 ; $(\mathrm{SE}=0.45)$ points]. There were significant differences between groups in final notes for physical activity knowledge. Simple educational activities produced positive effects on health eating knowledge however, for physical activity may be necessary longer intervention.

Keywords: Physical activity. Health eating. Education. School.

\section{Introdução}

O estilo de vida moderno, com baixos níveis de atividade física e consumo reduzido de frutas e verduras, associado ao elevado nível de sedentarismo e ingestão de alimentos industrializados de alto valor energético, tem resultado no aumento da prevalência das doenças crônicas não transmissíveis (DCNT) na população brasileira (MENDONÇA, 2004; WHO, 2004). As DCNT diminuem a expectativa e a qualidade de vida, além de afetar a capacidade de trabalho, representando um grande desafio social e econômico às sociedades modernas (BRASIL, 2006; WHO, 2004).

Os hábitos e a qualidade de vida estão ligados a diversos determinantes e condicionantes sociais, econômicos, culturais, políticos e fisiológicos (MENDONÇA, 2004). A Promoção da Saúde pode ser compreendida como a ação conjunta da população, de diversos setores produtivos da sociedade e do Estado com o objetivo da melhoria das condições de saúde individual e coletiva (OPAS, 1998); uma proposição bastante complexa e abrangente. Em termos de ações práticas, o estímulo ao desenvolvimento sustentável de estilos de vida saudáveis pode ser utilizado como um indicador de promoção da saúde que visa reduzir os fatores de risco para DCNT (BRASIL, 2006).

A escola, além de transmitir conhecimentos sobre a saúde organizados em diversas disciplinas, deve também educar e desenvolver valores relacionados ao estilo de vida e a posturas críticas diante da realidade social, em um processo de aquisição de competências que 
sustente as aprendizagens ao longo da vida e que promova autonomia e empoderamento na manutenção da saúde (BRASIL, 2009; HALLAL, 2010). O estímulo ao desenvolvimento de hábitos saudáveis durante a infância e a adolescência é relevante, pois uma vez estabelecidos nesta etapa da vida, têm grandes chances de serem perpetuados até a vida adulta (BRASIL, 2009; FIGUEIREDO et al., 2010).

A educação e informação sobre atividade física e alimentação saudável são formas de promover 0 desenvolvimento de atitudes positivas em saúde (BRASIL, 2009; WHO, 2004). Apesar de não haver comprovação da causalidade entre mudanças nos níveis de conhecimento e alterações de atitudes no estilo de vida (DOMINGUES et al.,2004; KNUTH et., 2009); alguns estudos mostram que o aumento do conhecimento sobre práticas alimentares tem associação com escolhas dietéticas mais saudáveis e diminuição dos índices de adiposidade em escolares (GAGLIANONE et al., 2006; TRICHES; GIUGLIANI, 2005; FISBERG et al., 2000).

Os professores representam um importante canal de comunicação e informação, pois apresentam similaridade comunicativa e estão envolvidos na realidade social, servindo de referência de conhecimentos e comportamentos para os alunos (BORGES et al., 2009; DE OLIVEIRA, 2010; DOYLE e FELDMAN, 1994). Independente das disciplinas que ministram, é fundamental que os professores possuam informações atualizadas sobre promoção da saúde e que utilizem este conteúdo em suas práticas pedagógicas de maneira transversal e interdisciplinar (DE OLIVEIRA, 2010; GAGLIANONE et al., 2006; HALLAL, 2010), estimulando 0 desenvolvimento de atitudes saudáveis entre os escolares (BRASIL, 2009).

Apesar de extremamente relevante e atual, pouco se sabe sobre o nível de conhecimento e as práticas pedagógicas dos professores em temas relacionados à promoção da saúde. Pouco se conhece também sobre os efeitos que diferentes intervenções educativas possuem nesse contexto, pois a falta de estudos e a diversidade de estratégias utilizadas nas intervenções contribuem para a existência desta lacuna no conhecimento. A produção de evidências científicas sobre quais os modelos interventivos mais eficientes para capacitar professores da rede pública de ensino em promoção da saúde pode favorecer o processo de aquisição de capacidades e desenvolvimento de estratégias neste importante tema.

Sendo assim, o objetivo deste estudo é avaliar os efeitos de aprendizagem gerados por intervenções educativas do tipo longa (IL) e curta (IC) nos conhecimentos sobre alimentação saudável e prática regular de atividade física em professores do ensino fundamental da rede pública de ensino.

\section{Métodos}

Este é um estudo quasi-experimental, aprovado pelo comitê de ética da Faculdade de Ciências da Saúde da Universidade de Brasília e desenvolvido no decorrer do ano de 2007 em Santa Maria, uma cidade satélite que abriga população de baixa renda na periferia do DF. Duas escolas foram selecionadas por conveniência entre as onze escolas de ensino fundamental de Santa Maria-DF, por estarem situadas próximas ao Núcleo de Extensão da Universidade, o que facilitou o desenvolvimento das intervenções educativas propostas no estudo.

Todos os professores dessas duas escolas foram convidados a participar da pesquisa, e nenhum professor se recusou a participar. A amostra final foi composta de 42 professores que, após assinarem o termo de consentimento livre e esclarecido, informaram seu sexo, idade e quantificaram bens de consumo familiar e o nível de educação formal do chefe da família; informações que foram utilizadas para classificar o nível sócio econômico através dos critérios de classificação econômica Brasil proposto pela Associação Brasileira de Empresas de Pesquisa (ABEP, 2003).

Durante os horários de coordenação os professores responderam individualmente aos questionários de conhecimentos sobre atividade física e alimentação saudável em relação com a promoção da saúde. O nível de conhecimento foi analisado a partir do somatório de pontos obtidos nos questionários, com pontuação mínima de $0 \mathrm{e}$ máxima de 10 pontos. Cada questão recebeu pesos diferentes de acordo com sua relevância e complexidade (Apêndice 01).

Os questionários foram desenvolvidos para este estudo com a elaboração de seis questões 
baseadas nas recomendações de alimentação saudável (Philippi et al, 1999; WHO, 2003) e seis questões sobre a prática de atividade física ( $\underline{\text { ACSM }}, 1998 ; \underline{\text { WHO }}$, 2002) para promoção da saúde e prevenção de DCNT. Os questionários foram testados em estudantes de educação física e nutrição e correções foram feitas para facilitar o entendimento das questões e otimizar o tempo de aplicação, entretanto, não foi feito um teste de confiabilidade. A pontuação de cada resposta foi definida de acordo com a proximidade da resposta ideal e em função da complexidade e relevância da questão; aspectos deliberados e definidos por uma equipe de três pesquisadores doutores da área. As questões específicas sobre as recomendações de atividade física e alimentação saudável para a prevenção de DCNT e promoção da saúde receberam peso maior por terem relação direta com o objeto do estudo.

Após o levantamento do nível de conhecimento inicial, os professores de cada escola receberam um dos dois modelos de intervenção educativa: a Intervenção Longa (IL; $n=31$ ) ou a Intervenção Curta (IC; $n=11$ ), cada uma com o uso de estratégias e recursos adequados ao período de duração. A diferença no número de sujeitos entre as intervenções refletiu a quantidade de professores que estavam presentes no(s) dia(s) da realização da pesquisa e ao diferente número de visitas à cada escola. Professores que estavam de licença médica ou em sala de aula no(s) horário(s) de visita não foram incluídos.

As duas intervenções forneceram material educativo impresso na forma de pirâmide colorida tridimensional e foram afixados 10 Banners em locais visíveis da escola com grande circulação de pessoas da comunidade escolar por um período de duas semanas. Em adição, a IL foi constituída de um curso de extensão de 40 horas realizado no decorrer de três meses com dois encontros presenciais por semana nos horários de planejamento pedagógico dos professores, com palestras, debates e leitura de textos. A IC foi constituída de uma palestra com duração de 30 minutos sobre o tema.

Todas as ações educativas contemplavam os temas de alimentação saudável e atividade física como fatores de promoção da saúde e prevenção de DCNT. Todos os professores foram encorajados a desenvolver atividades relacionadas aos temas da intervenção nas aulas das disciplinas sob sua responsabilidade.

Ao final do período de cada intervenção (IL, 3 meses; IC, 2 semanas), os professores responderam aos questionários de conhecimento pós-intervenção de maneira similar à descrita na fase pré-intervenção. Uma questão semiaberta avaliou a opinião dos professores sobre a qualidade das intervenções e uma questão dicotômica verificou se houve inclusão dos temas abordados durante as intervenções nas aulas ministradas pelos professores.

Dados descritivos da amostra foram reportados como porcentagem, média $(M)$, desvio padrão (DP) e erro padrão (EP). O efeito aprendizagem foi avaliado por meio da diferença entre a nota obtida no questionário pós-intervenção menos a nota no questionário pré-intervenção. A melhora do nível do conhecimento foi considerada quando o somatório de pontos pós-intervenção foi maior que o do pré-intervenção.

Em seguida utilizou-se o teste $t$-Student para grupos independentes para comparar as médias do efeito da aprendizagem com o intuito de verificar diferenças por tipo de intervenção (longa ou curta), permitindo identificar qual o modelo de intervenção educativa apresentou melhores resultados. $O$ percentual de professores em cada grupo que melhorou seu nível de conhecimento e que utilizou os conhecimentos em suas práticas pedagógicas foi reportado e possíveis diferenças significativas entre os grupos foram investigadas através do teste Qui-Quadrado. A análise estatística foi realizada utilizando 0 programa SPSS versão 17.0 , e o nível de significância adotado foi de $95 \%(p<0,05)$.

Este estudo recebeu apoio financeiro do $\mathrm{CNPq}$ - Conselho Nacional de Desenvolvimento Científico e Tecnológico Edital MCT-CNPq $51 / 2005$ para reprodução do manual do entrevistador, material educativo e questionários; aquisição de computador para análise dos dados e de combustível para o transporte dos pesquisadores.

\section{Resultados}

A amostra foi composta de 42 professores de duas escolas de ensino fundamental de Santa 
Maria-DF, $59,5 \%$ do sexo feminino e $40,5 \%$ do sexo masculino, todos com nível superior completo e idade média (DP) de 38,8 $(1,2)$ anos. Em relação à renda familiar, $58,1 \%$ relatou ter renda entre 5 a 9 salários mínimos (SM); 32,6\% entre 10 a 20 SM; 4,7\% entre 2 a 4 SM; e 4,7\% superior a $20 \mathrm{SM}$; o SM à época da entrevista era $\mathrm{R} \$ 350,00$.

A nota média (DP) dos níveis iniciais (préintervenção) e finais (pós-intervenção) de conhecimento em atividade física e alimentação saudável, bem como a nota média (EP) do efeito aprendizagem, separado por tipo de intervenção é apresentada na tabela 1 . O nível de conhecimento inicial, aferido pré-intervenção, não variou significativamente em função da classe social, nível de escolaridade, sexo, nem grupo de intervenção.

Tabela 1. Média (M) e desvio padrão (DP) dos níveis iniciais (pré-intervenção) e finais (pós-intervenção) do conhecimento sobre atividade física (AF) e alimentação saudável (AS), e nota média (M) e erro padrão (EP) do efeito aprendizagem, separados por intervenção longa (IL) e curta (IC).

\begin{tabular}{ccccccc}
\hline & \multicolumn{4}{c}{ Nota AF } & \multicolumn{2}{c}{ Nota AS } \\
\cline { 2 - 7 } & Pré & Pós & $\begin{array}{c}\text { Efeito } \\
\text { aprendizagem }\end{array}$ & Pré & Pós & $\begin{array}{c}\text { Efeito } \\
\text { aprendizagem }\end{array}$ \\
\hline Intervenção & $\mathbf{M}(\mathrm{DP})$ & $\mathbf{M}(\mathrm{DP})$ & $\mathbf{M}(\mathrm{EP})$ & $\mathbf{M}(\mathrm{DP})$ & $\mathbf{M}(\mathrm{DP})$ & $\mathbf{M}(\mathrm{EP})$ \\
IL $(\mathrm{n}=31)$ & $5,3(2,1)$ & $7,0(1,7)^{*}$ & $+1,69(0,52)$ & $6,5(1,6)$ & $7,8(1,0)^{*}$ & $+1,19(0,31)$ \\
IC $(\mathrm{n}=11)$ & $5,8(2,0)$ & $5,5(2,3)$ & $-0,36(0,45)$ & $6,4(1,2)$ & $7,5(1,2)^{*}$ & $+1,18(0,34)$ \\
\hline
\end{tabular}

${ }^{*}$ Diferença significativa com $\mathrm{p}<0,05$.

Após o término das intervenções, a avaliação do efeito aprendizagem nos conhecimentos sobre alimentação saudável não apresentou diferença entre os tipos de intervenção, entretanto, em relação à atividade física o conhecimento foi significativamente diferente $(p<0,05)$ entre os tipos de intervenção por meio do teste $t$ $(34,5)=2,97$, com estimativa de tamanho de efeito de intensidade média $r=0,45$. A comparação do efeito de aprendizagem por tipo de intervenção em média e intervalo de confiança do conhecimento em alimentação saudável e atividade física estão apresentados nos gráficos 1 e 2 respectivamente.

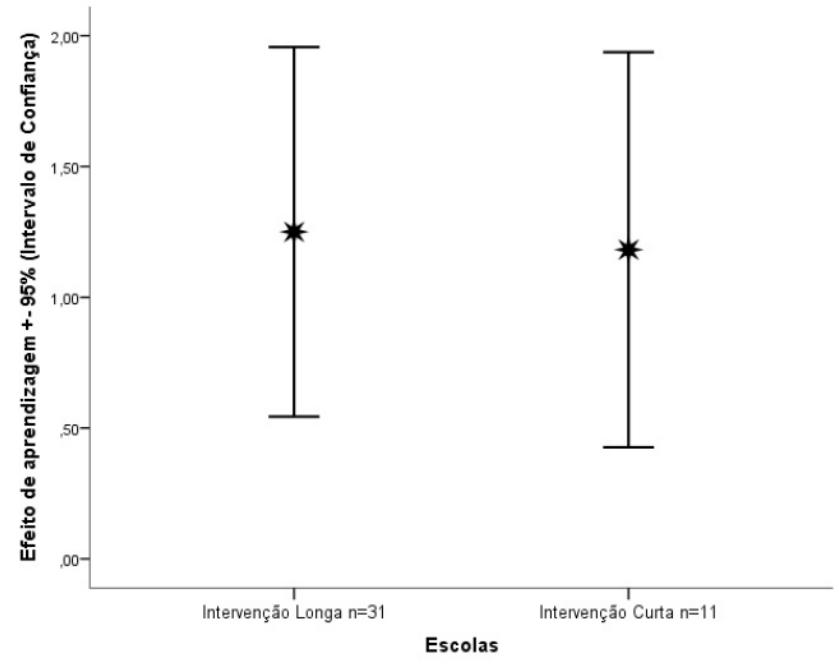

Grafico 1. Comparação entre as médias e intervalo de confiança do efeito de aprendizagem (nota pós- intervenção e nota pré-intervenção) do conhecimento em Alimentação Saudável separado por tipo de intervenção.

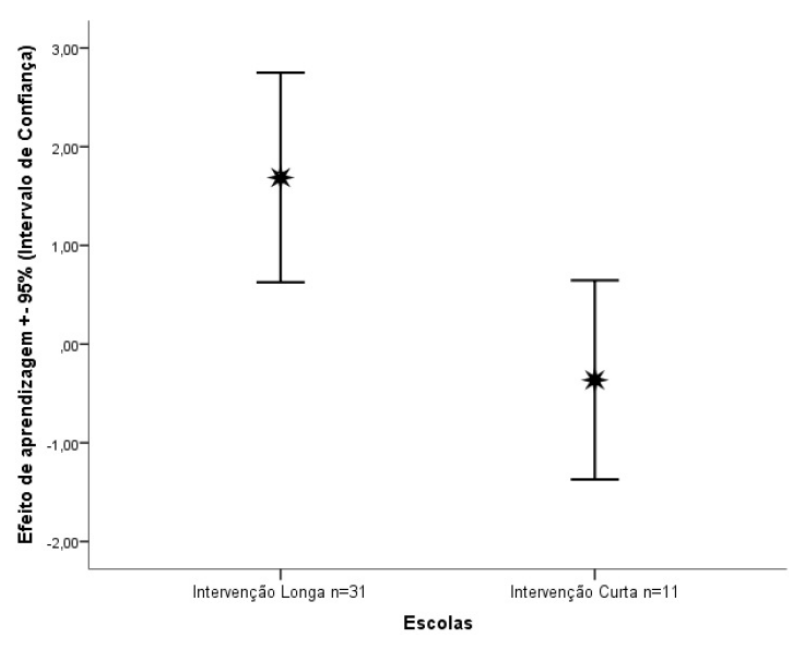

Grafico 2. Comparação entre as médias e intervalo de confiança do efeito de aprendizagem (nota pósintervenção e nota pré-intervenção) do conhecimento em Atividade Física separado por tipo de intervenção.

\section{Discussão}

A amostra estudada foi bastante homogênea, pois todos os professores participantes reportaram nível de escolaridade superior completo e a maioria foi classificada com nível sócio-econômico equivalente à classe média (ABEP, 2003). Estes dados refletem também a realidade do quadro de professores nas escolas do ensino público do DF ( 
Alguns estudos apontam que o nível de conhecimento em atividade física e alimentação saudável apresentam diferenças por renda econômica (BORGES et al., 2009; DOMINGUES et al., 2006; KNUTH et al., 2009), nível de escolaridade (BORGES et al., 2009; DOMINGUES et al., 2006; KNUTH et al., 2009) e sexo (DOMINGUES et al., 2006; WESTENHOEFER, 2005). Entretanto, este estudo não identificou a influência destes fatores no nível de conhecimento dos professores. Provavelmente por causa da homogeneidade da amostra e pelo fato de os conhecimentos sobre alimentação saudável e atividade física investigados neste estudo serem de caráter genérico, relacionados à promoção da saúde, logo, não são suscetíveis às influências socioculturais e às questões relacionadas ao sexo (DOMINGUES et al., 2006).

O conhecimento inicial sobre atividade física e alimentação saudável dos professores foi de nível intermediário e sem diferença significativa entre os sexos e entre as duas escolas. Ao considerar o papel do professor como educador e agente catalisador das ações de promoção da saúde na escola, fica evidente a necessidade de desenvolver suas capacidades e competências sobre o tema, para facilitar a capilarização das ações e conceitos em promoção da saúde para toda a comunidade escolar (HALLAL, 2010; TRICHES; GIUGLIANI, 2005; TORRES et al., 2009).

Intervenções educativas podem ter diversos formatos, estratégias e podem utilizar uma variedade de recursos materiais e humanos (TORRES et al., 2009). No presente estudo, as duas intervenções realizadas foram simples, de baixo custo, aproveitaram alunos de graduação e pós-graduação em formação para dinamizar 0 processo e produziram aumento na média dos níveis de conhecimento dos professores. Resultados similares foram apresentados em outros estudos (GAGLIANONE et al., 2006;

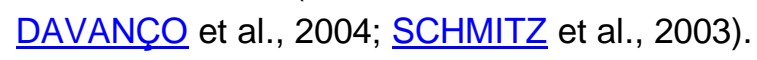

Estudo sobre professores de escolas públicas de São Paulo que receberem o curso de formação de 30 horas sobre alimentação saudável e sua importância na escola relata aumento no nível de conhecimento e de conscientização sobre seu papel no desenvolvimento de hábitos alimentares saudáveis de estudantes (GAGLIANONE et al., 2006). Outro estudo sobre professores de escolas públicas que também participaram de curso de extensão com 30 horas sobre nutrição, atividade física e saúde relata que o grupo experimental apresentou conhecimento significativamente maior do que os professores do grupo controle (DAVANÇO et al., 2004). Terceiro estudo de professores e cantineiros de escolas públicas e privadas que receberam intervenção educativa para o desenvolvimento de ambientes e práticas nutricionais saudáveis com duração entre 30 e 60 horas, relata aumento significativo do nível de conhecimento (SCHMITZ et al., 2008). Não foram encontrados outros estudos com professores sobre intervenções educativas e conhecimentos em atividade física e saúde no Brasil.

O presente estudo encontrou menores níveis iniciais de conhecimento em atividade física do que em alimentação saudável. Melhoras na média do nível de conhecimento em atividade física ocorreram apenas na IL. A complexidade que permeia a recomendação para promoção da atividade física e saúde proposta pela American College Sports Medicine, que inclui intensidade, frequência e duração da atividade, pode dificultar o entendimento e o ganho de conhecimento neste conteúdo (ACSM, 1998). É provável que a compreensão e fixação de conhecimentos relacionados à atividade física e saúde necessitem de períodos maiores de intervenção e da utilização de estratégias que favoreçam o debate e o esclarecimento de dúvidas como, por exemplo, as utilizadas durante a IL. Entretanto, estes resultados devem ser entendidos como indicadores, pois os questionários utilizados não foram validados ou tiveram sua confiabilidade testada.

Cabe ressaltar que a escola que recebeu a IL teve um número maior de participantes envolvidos na intervenção educativa do que a escola que recebeu a IC, que teve a quantidade de participantes limitada aos professores presentes no dia da realização da palestra. Por outro lado, na IL, $37,5 \%$ dos professores não cumpriram o mínimo de $75 \%$ das atividades propostas pela intervenção, critério utilizado para a emissão de certificação no curso de extensão. Para evitar que a evasão caracterizasse um viés de mortalidade experimental a análise dos efeitos da IL considerou todos os professores. A menor frequência observada nas intervenções foi de 
$65 \%$ e não foi encontrada diferença nas notas de conhecimentos entre professores que receberam o certificado e aqueles que não cumpriram o critério mínimo estabelecido para receber o certificado, provavelmente porque a parte da carga horária que teve maior evasão estava relacionada à elaboração e realização de atividades na escola.

Outro estudo apresentou resultados similares com diminuição da adesão no decorrer da intervenção de 60 horas; onde $46 \%$ dos participantes não concluíram $70 \%$ do processo

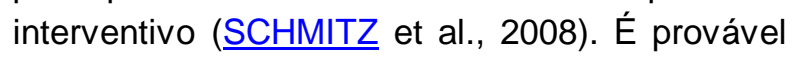
que períodos mais longos de intervenção possam favorecer o ganho de conhecimento e maior conscientização entre os professores, mas podem também reduzir sua adesão às intervenções (DAVANÇO et al., 2004).

O presente estudo não investigou mudanças nos hábitos de vida dos professores, mas identificou que um pouco mais da metade do grupo IL e quase metade do grupo IC apresentaram resultados positivos quanto à incorporação do conhecimento e aplicação em suas aulas. Este fato também foi observado em outro estudo, em que professores participantes da intervenção realizaram alguma atividade relacionada ao conteúdo da intervenção em sala de aula (DAVANCCO et al., 2004).

Diversos estudos apontam que o aumento do conhecimento sobre atividade física não necessariamente está relacionado com a adoção de um estilo de vida mais ativo (KNUTH et al., 2007; MARCONDELLI et al., 2008; MARCUS et al., 2006). Entretanto, abordagens educativas que visam modificar o estilo de vida com o aumento dos níveis de atividade física possuem como aspecto principal $o$ conhecimento $e$ a conscientização sobre a importância da atividade física para a promoção da saúde (MARCUS et al., 2006). Já estudos sobre alimentação saudável mostram que maiores níveis de conhecimento nutricional têm sido associados ao aumento no consumo de frutas e verduras (FISBERG et al., 2000; TRICHES; GIUGLIANI, 2005; WARDLE et al., 2000).

As intervenções simples e de baixo custo realizadas neste estudo, em especial a IL, resultaram no aumento do conhecimento dos professores nos temas de atividade física, alimentação saudável e promoção da saúde. Não foi possível determinar o grau de empoderamento e autonomia adquiridos, bem como não é possível afirmar que estas mudanças permanecerão a longo prazo, e quais os efeitos que este conhecimento ampliado terá na saúde e qualidade de vida dos professores e dos escolares. Entretanto sabe-se que 0 desenvolvimento de projetos que trabalham a autonomia do sujeito e a co-responsabilidade indivíduo-sociedade na manutenção da saúde podem gerar mudanças efetivas e duradouras nas escolhas e hábitos de vida (BRASIL, 2006; GAGLIANONE et al., 2006).

Alguns estudos mostram que os professores têm papel protagonista na realização de intervenções educativas na escola gerando mudanças positivas segundo Gaglianone et al., (2006) na alimentação, e de acordo com Mahar et al., (2006) nos hábitos de atividade física entre os alunos. Para Vargas et. al, (2011) o envolvimento de professores poderia favorecer a obtenção de resultados mais expressivos e contribuir para a continuidade das ações interventivas que foram realizadas com estudantes do $5^{\circ}$ e $6^{\circ}$ ano do ensino fundamental por um período de 4 meses nos temas de educação nutricional e incentivo a prática de atividade física. Os estudantes apresentaram resultados positivos na manutenção do consumo de frutas e hortaliças, mas não houve redução expressiva no consumo de refrigerantes.

Algumas limitações do presente estudo foram: a) a seleção da amostra foi não-probabilística, por conveniência e sem cálculo amostral o que não permite considerar a amostra estudada como representativa da população de professores da rede pública do Distrito Federal, apesar das similaridades de nível socioeconômico e escolaridade apresentadas; b) a dificuldade em elaborar um questionário que refletisse a abrangência do tema "atividade física, alimentação saudável e saúde" foi suplantada, ao menos parcialmente, ao incluir uma questão que valia o maior número de pontos sobre as recomendações divulgadas como referência em promoção da saúde por organismos internacionais; c) a utilização de questionário não validado limita a análise do nível de conhecimento dos professores e sua comparação com outros estudos mas não impede a avaliação da mudança no nível de conhecimento pela comparação dos resultados obtidos antes e após 
a intervenção; d) o aumento de conhecimento foi medido logo após cessar o período de intervenção, não sendo possível afirmar que os professores manterão níveis elevados de conhecimento ou práticas pedagógicas de promoção da saúde após períodos mais longos sem intervenções. Entretanto, cabe ressaltar que a coleta de dados após a intervenção ocorreu no final do semestre letivo, período em que os professores estão mais atarefados com correção de provas, trabalhos e fechamento de notas sendo que, se a coleta fosse realizada em um período diferente do ano, os professores poderiam apresentar melhores resultados.

Em síntese, o presente estudo aponta que professores de ensino fundamental apresentam nível mediano de conhecimento em atividade física, alimentação saudável e promoção da saúde, e que ações educativas simples e de baixo custo produziram efeitos positivos no conhecimento como conteúdo transversal nas aulas. A definição da estratégia interventiva a ser empregada deve ser decidida levando-se em conta questões como a complexidade do conteúdo a ser repassado e possíveis problemas com adesão. Aumentar o conhecimento pode ser - primeiro passo para motivar ações de promoção da saúde, resultando em benefícios pessoais, sociais e econômicos a todos os envolvidos na comunidade escolar.

\section{Referências}

AMERICAN COLLEGE OF SPORTS MEDICINE POSITION STAND. The Recommended Quantity and Quality of Exercise For Developing and Maintaining Cardiorespiratory an Muscular Fitness, and Flexibility In Healthy Adults. Medicine and science in sports and exercise . Indianapolis, v. 30, p. 975-91, 1998.

ASSOCIAÇÃO BRASILEIRA DE EMPRESAS DE PESQUISA (ABEP). 2003; Disponível em: http://www.abep.org/codigosguias/ABEP CCEB.p df. Acesso: 08 out. 2007.

BORGES, T. T. et al . Conhecimento sobre fatores de risco para doenças crônicas: estudo de base populacional. Cadernos de Saúde Pública, Rio de Janeiro, v. 25, n. 7, July 2009.

Disponível em:

http://www.scielo.br/scielo.php?script=sci arttext\& pid $=$ S0102-

311X2009000700009\&Ing=en\&nrm=iso. Acesso: 08 mar. 2011.
BRASIL. Ministério da Saúde. Secretaria de Vigilância em Saúde. Política Nacional de Promoção da Saúde/ Ministério da Saúde, Secretaria de Atenção a Saúde. Brasília: Ministério da Saúde, 2006.

BRASIL. Ministério da Saúde. Secretaria de Atenção a Saúde. Departamento de Atenção Básica. Cadernos de Atenção Básica. Saúde na escola. Série B. Textos Básicos de Saúde Cadernos de Atenção Básica, n. 24. Brasília, $1^{\circ}$ edição 2009.

DAVANCO, G. M. et al. Conhecimentos, atitudes e práticas de professores de ciclo básico, expostos e não expostos a Curso de Educação Nutricional. Revista de Nutrição, Campinas, v. 17, n. 2, June 2004. Disponível em: $<$ http://www.scielo.br/scielo.php?script=sci arttext \&pid $=S 1415-$

$52732004000200004 \& \operatorname{lng}=e n \& n r m=i s 0>$. Acesso: 08 mai. 2011.

DE OLIVEIRA, L.. Promoção da saúde na Educação Física Escolar: concepções e propostas na perspectiva de Professores do Ensino Público Estadual da Região Centro-Oeste do Município de São Paulo. Motriz. Revista de Educação Física. UNESP, Rio Claro, 16, fev. 2010. Disponível em:

http://www.periodicos.rc.biblioteca.unesp.br/index .php/motriz/article/view/1980-

6574.2010v16n2p535/3027 Acesso: 02 jun. 2011.

DOMINGUES, M. R.; ARAUJO, C. L. P. Conhecimento e percepção sobre exercício físico em uma população adulta urbana do sul do Brasil. Cadernos de Saúde Pública, Rio de Janeiro, v. 20, n. 1, Feb. 2004. Disponível em: http://www.scielosp.org/scielo.php?script=sci artt ext\&pid=S0102-

$311 \times 2004000100037 \&$ Ing =en\&nrm=iso Acesso: 08 mai. 2011.

DOYLE, E.I.; FELDMAN, R.H.L. Are local teachers or nutrition experts perceived as more effective among Brazilian high school students? J Journal of School Health, Kent OH, v. 64, n. 3, 1994.

FIGUEIREDO, T. A. M.; MACHADO, V. L. T. Representações sociais da homeopatia: uma revisão de estudos produzidos no Estado do Espírito Santo. Ciência e saúde coletiva, Rio de Janeiro, 2011. Disponível em:

http://www.scielo.br/scielo.php?script=sci arttext\& pid $=$ S1413-

$81232011000700032 \&$ Ing=en\&nrm=iso. Acesso: 09 jul. 2011. 
FISBERG, M. et al. Hábitos alimentares da adolescência. Pediatria Moderna. São Paulo, v. 36 n 11. 2000.

GAGLIANONE, C. P. et al . Nutrition education in public elementary schools of São Paulo, Brazil: the Reducing Risks of IIIness and Death in Adulthood project. Revista de Nutrição, Campinas, v. 19, n. 3, June 2006. Disponível em:

http://www.scielo.br/scielo.php?script=sci arttext\& pid $=S 1415-$

52732006000300002\&lng=en\&nrm=iso. Acesso: 22 jul. 2011.

GOVERNO DO DISTRITO FEDERAL. SECRETARIA DE ESTADO DE EDUCAÇÃO DO DISTRITO FEDERAL. Relatório de gestão da secretaria de estado de educação do Distrito Federal 2008. Disponível em: www.se.df.gov.br/300/30003007.asp. Acesso: 17 abr. 2009.

HALLAL PC. Promoção da atividade física no Brasil: chegou a hora da escola. Revista Brasileira de Atividade Física e Saúde. Florianópolis, v. 15, n. 2, p.76-77, 2010.

KNUTH, A. G. et al. Conhecimento de adultos sobre o papel da atividade física na prevenção e tratamento de diabetes e hipertensão: estudo de base populacional no Sul do Brasil. Cadernos de Saúde Pública, Rio de Janeiro, v. 25, n. 3, Mar. 2009. Disponível em:

http://www.scielosp.org/scielo.php?script=sci artt ext\&pid=S0102-

311X2009000300006\&Ing=en\&nrm=iso_Acesso: 02 jun. 2011.

MAHAR, M.T; MURPHY, S.K et al. Effects of a classroom-based program on physical activity and on-task behavior. Medicine and science in sports and exercise. Indianapolis, v. 38 n. 12, 2006. DOI:

10.1249/01.mss.0000235359.16685.a3.

MARCONDELLI, P; COSTA, T.H.M; SCHMITZ, B.A.S. Nível de atividade física e hábitos

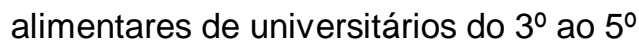
semestres da área da saúde. Revista de Nutr., Campinas, v. 21, n. 1, Feb. 2008. Disponível em: http://www.scielo.br/scielo.php?pid=S141552732008000100005\&script=sci arttext Acesso: 22 ago. 2011.

MARCUS, B.H; WILLIAMS, D.M; DUBBERT, P.M et al. Physical activity intervention studies: what we know and what we need to know: a scientific statement from the American Heart Association Council on Nutrition, Physical Activity, and Metabolism (Subcommittee on Physical Activity); Council on Cardiovascular Disease in the Young; and the Interdisciplinary Working Group on Quality of Care and Outcomes Research. Circulation. 2006.

http://faculty.ksu.edu.sa/hazzaa/Resources/Physi cal\%20Activity\%20Intervention\%20Studies\%20\%20Circulation\%202006.pdf . Acesso: 16 mai. 2011.

MENDONCA, C. P.; ANJOS, L. A. Aspectos das práticas alimentares e da atividade física como determinantes do crescimento do sobrepeso/obesidade no Brasil. Cadernos de Saúde Pública, Rio de Janeiro, v. 20, n. 3, June 2004. Disponível em: http://www.scielo.br/scielo.php?script=sci arttext\& pid=S0102-

311X2004000300006\&lng=en\&nrm=iso. Acesso: 18 jun. 2011.

OPAS 1998. La Salud in las Americas, vol 1. OPAS, Washington. 386 pp.

PHILIPPI, S. T.; LATTERZA, A. R.; CRUZ, A. T.; Ribeiro LC. Pirâmide alimentar adaptada: guia para escolha dos alimentos. Revista de Nutrição, Campinas, 1999; 12:65-80.

SCHMITZ, B. A. S. et al . A escola promovendo hábitos alimentares saudáveis: uma proposta metodológica de capacitação para educadores e donos de cantina escolar. Cadernos de Saúde Pública, Rio de Janeiro, 2011. Disponível em: http://www.scielo.br/scielo.php?script=sci arttext\& pid=S0102311X2008001400016\&Ing=en\&nrm=iso . Acesso: 11 mai. 2011.

TORRES, Heloisa de Carvalho et al . Avaliação estratégica de educação em grupo e individual no programa educativo em diabetes. Revista Saúde Pública, São Paulo, v. 43, n. 2, Apr. 2009.

Disponível em:

http://www.scielosp.org/scielo.php?script=sci artt ext\&pid=S0034-

89102009000200010\&lng=en\&nrm=iso . Acesso: 16 ago. 2011.

TRICHES, R. M.; GIUGLIANI, E. R. J. Obesidade, práticas alimentares e conhecimentos de nutrição em escolares. Revista Saúde Pública, São Paulo, v. 39, n. 4, Aug. 2005. Disponível em: http://www.scielo.br/scielo.php?script=sci arttext\& pid=S0034-

$89102005000400004 \&$ Ing=en\&nrm=iso. Acesso: 19 mai. 2011.

VARGAS, Izabel Cristina da Silva et al . Avaliação de programa de prevenção de obesidade em adolescentes de escolas públicas. Revista Saúde Pública, São Paulo, v. 
45, n. 1, Feb. 2011. Disponível em:

http://www.scielo.br/scielo.php?script=sci arttext\& pid $=$ S0034-

89102011000100007\&lng=en\&nrm=iso. Acesso:

21 mar. 2011.

WESTENHOEFER $\mathrm{j}$. age and gender dependent profile and food choice. Forum of Nutrition. v.

57, 2005.

WARDLE, J; PARMENTER, K; WALLER, J.

Nutrition knowledge and food intake. Appetite. $v$. 34, 2000.

WHO. World Health Organization. Global strategy on diet, physical activity and health. Geneva: WHO, 2004.

WHO. World health Organization. Diet, nutrition and the prevention of chronic diseases.

Report of a Joint WHO/FAO. Expert Consultation. Geneva: World Health Organization. [WHO Technical Report Series, 916], 2003. Disponível em: http://www.who.int/dietphysicalactivity/en/. Acesso em 12 fev. 2008.

WHO. World Health Organization: Reducing risks, promoting healthy life. World Health Report 2002. Geneva: World Health Organization; 2002.

Agradecimento: Apoio financeiro do $\mathrm{CNPq}-$ Conselho Nacional de Desenvolvimento Científico e Tecnológico Edital MCT-CNPq $51 / 2005$.

\section{Endereço:}

Júlia Aparecida Devidé Nogueira

Universidade de Brasília - Campus Universitário

Darcy Ribeiro, Faculdade de Educação Física

Brasília DF Brasil

71000-000

Telefone: (61) 3107-2535

e-mail: julianogueira@unb.br

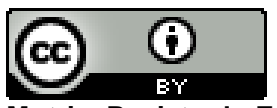

Motriz. Revista de Educação Física. UNESP, Rio Claro, SP, Brasil - elSSN: 1980-6574 - está licenciada sob Creative Commons - Atribuição 3.0 